\title{
Localised Primary Kaposi's sarcoma Of the Scrotum: A Rare Presentation.
}

\author{
Usman Mohammed Tela ${ }^{1}$ FWACS, Ahmed Gadam Ibrahim ${ }^{2}$ FWACS, \\ Adamu Sadiq Abubakar ${ }^{3} F M C A$, Baba WaruGoni ${ }^{4} F W A C P$, \\ Alhaji Bukar Musa ${ }^{5}$ M.Sc, AbdulmalikWaziri ${ }^{6} M B B S$ \\ ${ }^{I}$ Department of Surgery, University of Maiduguri Teaching Hospital, Nigeria. \\ ${ }^{2}$ Department of Surgery, University of Maiduguri Teaching Hospital, Nigeria. \\ ${ }^{3}$ Department of Anaesthesia and Intensive Care, University of Maiduguri Teaching Hospital \\ ${ }^{4}$ Department of Medicine, University of Maiduguri Teaching Hospital, Nigeria. \\ ${ }^{5}$ Department of Histopathology, University of Maiduguri Teaching Hospital, Nigeria. \\ ${ }^{6}$ Department of Surgery, University of Maiduguri Teaching Hospital, Nigeria.
}

\begin{abstract}
Kaposi's sarcoma is a malignancy of vascular origin that commonly presents with multifocal lesions affecting the skin, and other organs. Localized lesion involving the scrotal skin alone is quiet uncommon in the literature. We present a 48-year-old HIV-infected man with a localized scrotal lesion.He was evaluated and subsequently had total scrotectomy. The testes were free of tumor, and hence preserved. Post-operative outcome was satisfactory. High index of clinical suspicion is required for early diagnosis.
\end{abstract}

Keywords: Kaposi's sarcoma, Scrotal skin, HIV/AIDS

\section{Introduction}

Kaposi's sarcoma (KS) is a vascular lesion of low-grade malignant potential that presents most frequently with skin lesions ${ }^{1}$.A Hungarian Dermatologist Moritz Kaposi first described it in 1872. It usually presents as multifocal lesion, affecting the skin at any part of the body and or the viscera. In the generalised type of lesion, associated genitalia involvement has been described, but exclusive genitalia involvement is rare. ${ }^{2}$ Isolated involvement of the scrotum is even rarer. Very few case of such type of localised primary scrotallesions were reported in the literature. ${ }^{3,4}$ Kaposi's sarcoma is commonly seen among immunosuppressed patients especially HIV/AIDS.

Case report

A 47-year-old HIV-infected man presented with a progressive, painless, multi-nodular scrotal skin swelling of one-year duration. Three-month prior to presentation, the swelling rapidly increased in size, ulcerated spontaneously and started discharging offensive foul smelling fluid. There were no swellings in other parts of the body. He had no other genito-urinary, gastrointestinal or other systemic symptoms.He had been on highly active anti-retroviral therapy (HAART-Lamuvidine, Zidovudine and Nevirapine) 4 years prior to presentation. In addition, he had one-year history of erectile dysfunction.

On clinical examination he was found to be a middle-aged man with atheletic physique, calm, afebrile, not pale, anicteric no pedal oedema. Examination of the external genitalia revealed foul smelling, isolated multiple ulcerated nodular tumors with raised edges, involving the oedematous scrotum (fig. 1) and sparing the testes, penis and the perineum as shown in figure 2.There were multiple right inguinal lymphadenopathy.The rest of the abdomen, chest and other systems were normal. A working diagnosis of Dermato-fibrosarcoma to rule out Kaposi's sarcoma in a HIV-infected patient was made. Investigations done included wound swab for culture, which yielded significant growth of Klebsiella species and Staphylococcus aureus. Histology for the biopsy of the lesion showed Kaposi sarcoma of nodular variant with excision free margin. His CD4 count was $239 \mathrm{cells} / \mathrm{mm}^{3}$. Other investigations were,Hemoglobin concentration, serum electrolytes, urea and creatinine, abdomino-pelvic ultrasound scan and chest x-ray which were all normal. The patient was counseled and had total scrotectomy under spinal anesthesia; the testes were not involved by the tumor, and hence bilateral orchidopexy done in tunnels created on the medial side of upper part of both thighs as shown in figures 1a, b, c, and $\mathrm{d}$. Post-operative condition was satisfactory, and referred to the retroviral disease clinic. He was seen 6 month after the surgery with no evidence of recurrence. 


\section{Discussion}

Kaposi's sarcoma is one of commonest malignancies seen among HIV/AIDS patients. It usually presents as multifocal lesions involving the skin, lymph node, viscera, or mucosa. KS is also frequently reported among HIV-infected MSM (Male having Sex with Male) patients in the western world ${ }^{5}$.Concurrent opportunistic infection can occur in these types of patients.

Isolated scrotal lesion as in the index case presented is extremely rare in the literature. Combined lesion of the penis and other genital organ ${ }^{6}$, also involvement of genital $\operatorname{tract}^{7}$ were reported in the past. Generally, KS has been strongly linked with infection caused by Kaposi's sarcoma - associated Herpes virus [KSHV] also known as Human Herpes Virus type 8, but there is no clear aetiology of isolated lesion of the scrotum. In HIV-seropositive patients, the incubation period for diseases caused by KSHV infection largely depends on the host's immune status rather than on the duration of KSHV infection. The incidence of KS among AIDS patients is variable; depending on the region and the sub-population of patients ${ }^{8}$. Most of the primary KSHV infections appear to be asymptomatic. Not every patient with HIV/AIDS develops KS. There is variation in the multi-focality of KS among those who developed the lesions; this is determined by the strength of the immunity of the individual. There are basically 4 clinical types of KS. The commonest type is the Epidemic or AIDS associated type, similar to this case. The second type is classical KS; commonly seen among Ashkenazi Jews in Eastern Europe and the Mediterranean region; here it presents as indolent multiple purpleblue plaques on the hands and feet later progresses to the arms and legs. Thirdly, Endemic type of KS is commonly seen among HIV-negative Africans, especially in Uganda constituting 3 to 9\% of all cancers and also in Zambia. Lastly, the immunosuppressant-Associated or Transplantation-Associated KS is commonly seen in those receiving immunosuppressive drugs due to organ transplantation or other conditions.

Morphological variants of KS lesions include patchy, plaque and nodular types. Moreover, histologic subtypes have been described in the past as anaplastic, telengiectatic and lymphedematous variants. Other newly described variants include hyperkeratotic, keloidal, micronodular, pyogenic granuloma-like, ecchymotic and intravascular $\mathrm{KS}^{1}$.Histology of our index case revealed the nodular variant as shown in figure 2.

The clinical and histological type of the lesion guides treatment options for KS. Systemic chemotherapy using vinblastine, interferon or paclitaxel, and local therapy with 5\% imiquimod cream has yielded results. Radiotherapy produces good result especially in the classic nodular type. Surgical excision of localized nodular lesions yields good results. This was the treatment we opted for our patient, because the lesion was quiet large and amenable for excision with tumor free margin. Furthermore, surgery was chosen because it would relieve the patient from the burden of ulcerated foul smelling tumor. Moreover, chemotherapy drugs and radiotherapy were not readily available at our center- even where they are available most patients in subSaharan cannot afford them.

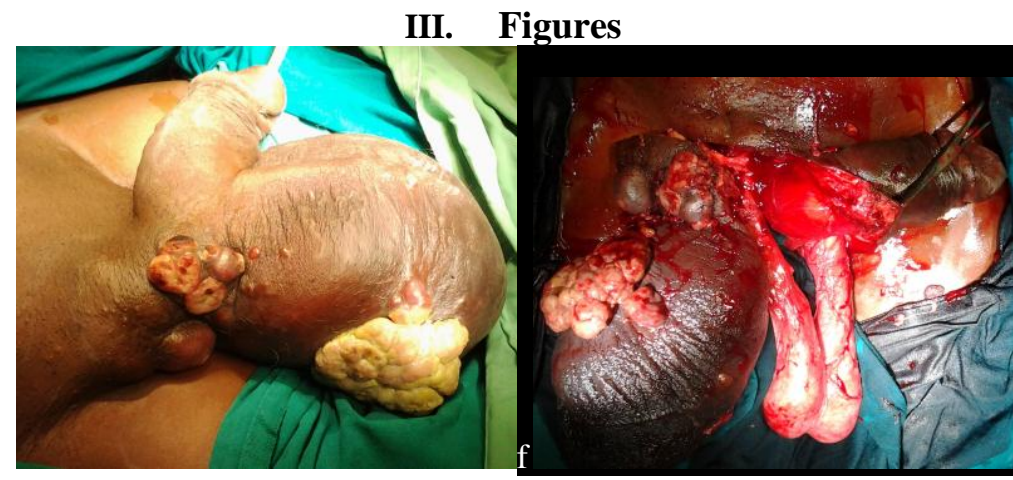

Figure 1a: scrotal Kaposi's sarcoma Figure 1b: Normal testes 


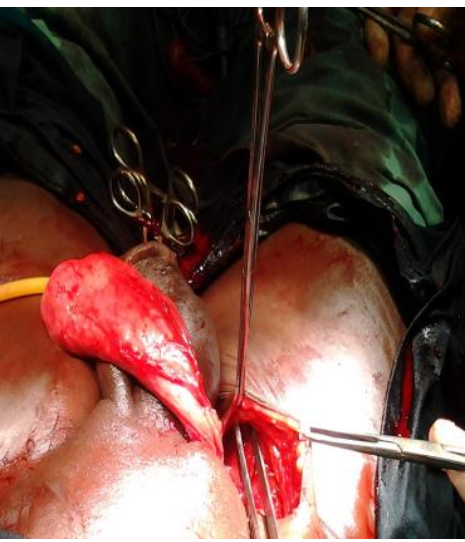

Figure 1c: tunnel for testes

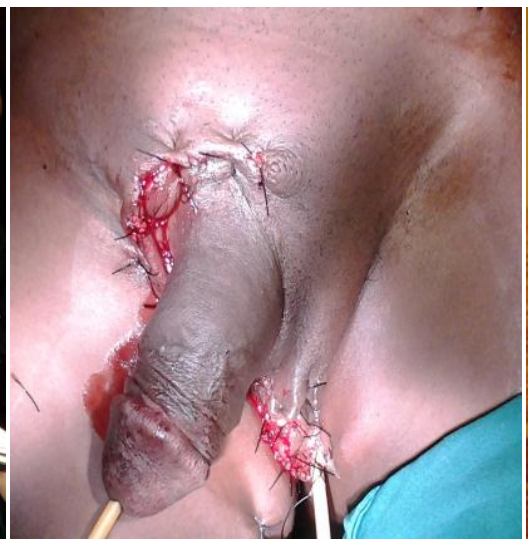

Figure1d: wound closed

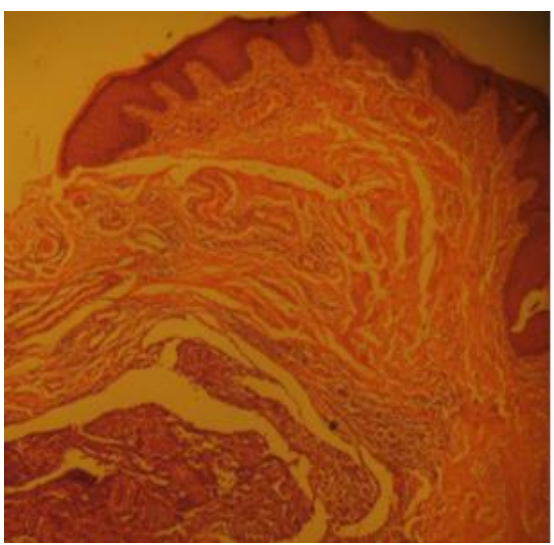

Figure: 2: Histology photomicrograh

\section{Conclusion}

Isolated Kaposi sarcoma of the scrotum is quiet rare. It presents with variable morphologic appearance, high index of clinical suspicion is necessary to achieve early diagnosis, especially among HIV-infected.

\section{Acknowledgement}

We wish to express our sincere appreciation to the Management of University of Maiduguri Teaching Hospital (UMTH), Nigeria and the staff of the department of surgery, UMTH, Nigeria. Our special thanks also go to the staff of department of histopathology and Harvard PEPFAR/Apin programme UMTH, Nigeria.

\section{References}

[1] Grayson W, Pantanowitz L, Histological variants of cutaneous Kaposi’ sarcoma. DiagnPathol 2008 Jul 25, 3:31

[2] Cox JW, Halprin K, Ackerman AB, Kaposi's sarcoma localized to the penis. Arch Dermatol 1970; 102:461-2

[3] Vyas S, ManabeT, Herman JR, Newman HR. Kaposi’s sarcoma of srotum. Urology, 1976 Jul; 8(1) 82:85

[4] Johnson DE, Chica J, Rodriquez LH, Luna M, Kaposi'ssarcoma presenting as scrotal lesions Urology. 1977 Jun; 9(6):686-688

[5] Friedman-Kien AE, Laubenstein L, Marmor M, et al. Kaposi's sarcoma and Pneumocystis pneumonia among homosexual men of New York City and California. MMWR Morb Mortal Wkly Rep 1981;30:305-308

[6] Bayne D, Wise GJ, Kaposi sarcoma of penis and genitalia: A disease of our times Urology, 1988.

[7] Linker D, Leiberman P, Grabstald H, Kaposi's sarcomaof genitourinary tract . Urology, 1975 May; 5(5):684-7Jan; 31(1):22-25.

[8] Kagu MB, Nggada HA, Garandawa HI, Askira BH, Durosinmi MA. AIDS-associated Kaposi's sarcoma in Northeastern Nigeria.Singapore Med J. 2006 Dec;47(12):1069-1074. 